\title{
Antibiotic Resistance Pattern of Bacteria Causing Hospital Acquired Infections in the New Mansoura General Hospital, Egypt
}

Rania Hassan ${ }^{1}$, Abdel-Hady El-Gilany², Amina M Abd Elaal ${ }^{3}$, Noha El-Mashad ${ }^{3}$ and Dalia Abdelazim $^{4}$

${ }^{1}$ Consultant of Clinical Pathology, Ministry of Health, Egypt

${ }^{2}$ Professor of Public Health \& Preventive Medicine, Faculty of Medicine, Mansoura University, Egypt

${ }^{3}$ Professor of Clinical Pathology, Faculty of Medicine, Mansoura University, Egypt

${ }^{4}$ Director of Molecular Genetics, Department of Pathology, NYU Winthrop University Hospital, USA

\begin{abstract}
Background: This study aims to describe the antibiotic resistance pattern among patients with hospital acquired infections in Mansoura New General Hospital (MNGH), Egypt and to identify the multidrug resistant organisms.

Methods: A descriptive study was carried out in different departments of MNGH. All the 272 microbiological samples (blood, urine, swabs and others) from 259 patients with hospital acquired infections during the study period were processed in the bacteriology laboratory on different culture media. Bacterial isolates were identified by Gram-stain, cultures on routine media (e.g Blood agar, MacConkey agar) and specific biochemical tests following standard procedures. Antibiotic susceptibility testing was performed using disk diffusion methods. Multidrug-resistant (MDR) was defined as isolate non-susceptible to at least one agent in more than three antimicrobial categories.

Results: The prevalence of methicillin-resistant Staphylocoocus aureus spp. (S. aureus) (MRSA) was 55.8\%, methicillinresistant Coagulase-negative (CoNS) was 100\%, and vancomycin-resistant Enterococcus spp. (VRE) was 20\%. Carbapenem resistant E. coli was $8.2 \%$. Cephalosporin resistant (CephRy-Klebsiella spp.) was $85 \%$, and carbapenem resistant $\mathrm{K}$. pneumoniae spp. was 21.6. MDR-P. aeruginosa was $43.6 \%$ and MDR A. baumannii spp. was $100 \%$.

Conclusion: There is a need to integrate testing bacteria for antibiotics sensitivity in all nosocomial infections surveillance system. Policies on the control of antibiotic usage have to be implemented to avoid the emergence of newer generations of resistant pathogens.
\end{abstract}

\section{Keywords}

Hospital acquired infections, Antibiotic resistant, Multidrug resistant organism

\section{Introduction}

The emergence of antimicrobial resistance (AMR) is a global public health problem, particularly for pathogens causing nosocomial infections. Rates of AMR are increasing rapidly and resistance patterns show large variations between countries [1]. Infections caused by resistant pathogens result in significant morbidity and mortality, and contribute to increase healthcare costs worldwide. Despite the availability of newer antibiotics, emerging antimicrobial resistance has become an increasing problem in many pathogens throughout the world [2].

The hospital is an epicenter for colonization and infection by drug resistant pathogens due to the frequent usage of potent antimicrobial agents in severely ill or immune compromised patients and suitable circumstances for patients to acquire resistant organisms, from reservoirs such as oth-

*Corresponding author: Abdel-Hady El-Gilany, Professor of Public Health \& Preventive Medicine, Faculty of Medicine, Mansoura University, Mansoura 35516, Egypt, Tel: 0106-071-4481

Accepted: February 13, 2021

Published online: February 15, 2021

Citation: Hassan R, Gilany AHE, Elaal AMA, et al. (2021) Antibiotic Resistance Pattern of Bacteria Causing Hospital Acquired Infections in the New Mansoura General Hospital, Egypt. Arch Community Med 3(1):16-21 
Citation: Hassan R, Gilany AHE, Elaal AMA, et al. (2021) Antibiotic Resistance Pattern of Bacteria Causing Hospital Acquired Infections in the New Mansoura General Hospital, Egypt. Arch Community Med 3(1):16-21

er patients, the environment, shared equipment, or hospital personnel [3].

Despite the presence of few studies in Egypt on antibiotic resistance none was done in Mansoura New General Hospital (MNGH). The objectives of this study are to describe the antibiotic resistance pattern among patients with nosocomial infections in MNGH. Egypt and to identify multidrug resistant organisms.

\section{Materials and Methods}

This descriptive study was done in MNGH, Egypt from $1 / 1 / 2017$ to $31 / 12 / 2017$. MNGH is a tertiary care hospital with 400 beds; it serves the Delta region of Egypt. It has 5 intensive care units (ICU) with 57 beds [general ICU (16 beds), Neonatal intensive care unit (NICU) (20 incubator), Pediatric intensive care unit (PICU) (6 beds), Cardiology care unit (CCU) ( 8 beds) and a neurosurgery ICU ( 7 beds)]. It has different internal medicine departments as General Internal Medicine, Nephrology, Neuromedecine, Pediatrics, and different surgical departments as General Surgery, Orthopedics, Neurosurgery, Ear, Nose and Throat, Obstetrics \& Gynecology, Urology, Vascular, Cardiothoracic, and Maxillofacial.

Every patient admitted to the hospital during the study period was monitored daily for developing nosocomial infections using standard definitions described by CDC [4]. A total of 272 microbiological specimens ( 87 blood, 83 urine, 61 surgical site swabs, 30 bronchial lavage/aspirate and 11 others) from 259 patients were processed in the bacteriology laboratory where they were isolated on different cultures. Bacterial isolates were identified by Gram-stain, cultures on routine media (e.g Blood agar, MacConkey agar) and specific biochemical tests following standard procedures. Antibiotic susceptibility testing was performed using disk diffusion methods and the result was interpreted according to the Clinical and Laboratory Standards Institute (CLSI) [5].

Multidrug-resistance (MDR) was defined according to CDC [6] as isolates which are non-susceptible to at least one agent in more than three antimicrobial categories. Specifically, an isolate of $A$. baymannii spp. was defined as having multidrug resistance (MDR) if it tested non-susceptible (i.e., resistant or intermediate) to at least one drug in three of the following six antimicrobial agents/groups: piperacillin or piperacillin/ tazobactam, extended-spectrum cephalosporins (cefepime or ceftazidime), aminoglycosides, ampicillin/sulbactam, carbapenems, and fluoroquinolones. For Pseudomonas aeruginosa spp. ( $P$. aeruginosa) isolates, MDR was defined as testing non-susceptible (i.e., either resistant or intermediate) to at least one drug in three of the five following antimicrobial groups: piperacillin or piperacillin/tazobactam, extended-spectrum cephalosporins (cefepime or ceftazidime), fluoroquinolones, aminoglycosides, and carbapenems.

Data was analyzed using SPSS program version 16 . Variables were presented as number and percent.

\section{Results}

The total number of isolated bacteria was 272 with $29.4 \%$ Gram positive bacteria (GPB) and $70.6 \%$ Gram negative bacteria (GNB). The most frequent GPB were $S$. aureus and $E n-$ terococcus spp. while the most frequent GNB were Klebsiella pneumoniae spp. (K. pneumoniae), E. coli spp. and P. aeruginosa spp. (Table 1).

The majority (74.4\%) of S. aureus spp. isolates were resistant to penicillin followed by resistance to cefoxitin (55.8\%). CoNS exhibits $100 \%$ resistance to cefoxitin and $75 \%$ resistance to penicillin. Enterococci spp. isolates were mostly resistant to ampicillin (88\%) and penicillin (80\%) (Table 2).

Third generation cephalosporin resistance was detected for different GNB. Resistance to ceftazidime was exhibited by $85 \%$ of $K$. pneumoniae spp., $71.8 \%$ of $P$. aeruginosa spp., $71.4 \%$ of $A$. baumannii spp., $69.7 \%$ E. coli spp. and $60.9 \%$ Proteus spp. Resistance to cefotaxime was exhibited by $85 \%$ of $K$. pneumoniae spp. $79.6 \%$ of E. coli spp. and $73.9 \%$ of Proteus spp., Resistance of E. coli spp., Proteus spp. and K. pneumoniae spp. to cefoperazone were $77.6 \%, 69.6 \%$, and $66.2 \%$; respectively. Resistance to the fourth generation cephalosporin (cefepime) was exhibited by $84.6 \%$ of $P$. aeruginosa spp.,

Table 1: Types of isolated pathogens.

\begin{tabular}{|c|c|c|c|}
\hline & Total isolates & \% of total isolates (272) & $\%$ of subtotal ${ }^{*}$ \\
\hline Gram positive bacteria & 80 & 29.4 & 100 \\
\hline S. aureus & 43 & 15.8 & 53.75 \\
\hline Enterococci & 25 & 9.2 & 31.25 \\
\hline CONS & 8 & 2.9 & 10.0 \\
\hline B hemolytic Streptococci & 4 & 1.5 & 5.0 \\
\hline Gram negative bacteria & 192 & 70.6 & 100 \\
\hline K. pneumoniae & 74 & 27.2 & 38.54 \\
\hline E. coli & 49 & 18.0 & 25.52 \\
\hline P. aeruginosa & 39 & 14.3 & 20.31 \\
\hline Proteus & 23 & 8.5 & 11.95 \\
\hline Acinetobacter & 7 & 2.6 & 3.65 \\
\hline
\end{tabular}

*Subtotal from either GPB or GNB 
Citation: Hassan R, Gilany AHE, Elaal AMA, et al. (2021) Antibiotic Resistance Pattern of Bacteria Causing Hospital Acquired Infections in the New Mansoura General Hospital, Egypt. Arch Community Med 3(1):16-21

Table 2: Antibiotic resistance pattern of isolated Gram positive organisms.

\begin{tabular}{|l|l|l|l|l|}
\hline & S. aureus & CoNS & Enterococci & B hemolytic Streptococci \\
& $(\mathbf{4 3})$ & $\mathbf{( 8 )}$ & $\mathbf{( 2 5 )}$ & (4) \\
\hline N (\%) & N (\%) & N (\%) & N (\%) \\
\hline Penicillin & NT & $6(75)$ & $20(80)$ & $1(25)$ \\
\hline Ampicillin & $24(55.8)$ & NT & $22(88)$ & $1(25)$ \\
\hline Cefoxitin & $16(39)^{*}$ & $3(50)^{*}$ & NT & NT \\
\hline Clindamycin & $15(36.6)^{*}$ & $3(37.5)^{*}$ & $15(60)$ & $2(50)$ \\
\hline Erythromycin & $6(13.9)$ & $1(12.5)$ & $5(20)$ & $1(25)$ \\
\hline Vancomycin & $10(23.3)$ & $1(12.5)$ & $4(16)$ & 0 \\
\hline Linezolid & $8(18.6)$ & $2(25)$ & $17(68)$ & 0 \\
\hline Levofloxacin & $9(20.9)$ & $2(25)$ & $17(68)$ & 0 \\
\hline Gentamicin & $18(41.9)$ & $3(37.5)$ & NT & NT \\
\hline Amikacin & NT & NT & $6(24)$ & NT \\
\hline Chloramphenicol & NT) & 0 \\
\hline
\end{tabular}

NT: Not tested; *Not tested with urine (2 samples); Nitrofurantoin tested only with urine samples with \% resistant to both Staphylococcus aureus and CoNS (50\%), and with Enterococcus resistant (83.3\%), Norfloxacin tested only with urine with \% resistant to Enterococcus (66.7\%)

Table 3: Antibiotic resistance pattern of isolated Gram negative organisms.

\begin{tabular}{|c|c|c|c|c|c|}
\hline & K. pneumoniae (74) & E. coli (49) & Proteus (23) & P. aeruginosa (39) & Acinetobacter (7) \\
\hline & $\mathrm{N}(\%)$ & $N(\%)$ & $\mathrm{N}(\%)$ & $\mathrm{N}(\%)$ & $N(\%)$ \\
\hline Ampicillin & $64(86.5)$ & $42(85.7)$ & NT & NT & NT \\
\hline Ampicillin/sulbactam & $61(82.4)$ & $29(59.2)$ & $20(87)$ & NT & $5(71.4)$ \\
\hline Piperacillin/Tazobactam & $62(83.8)$ & $30(61.2)$ & $9(39)$ & $22(56.4)$ & $7(100)$ \\
\hline Cefazolin & $65(87.5)$ & 46 (93.9) & $21(91.3)$ & NT & NT \\
\hline Ceftazidime & $63(85)$ & $34(69.7)$ & $14(60.9)$ & $28(71.8)$ & $5(71.4)$ \\
\hline Cefotaxime & $63(85)$ & 39 (79.6) & 17 (73.9) & NT & NT \\
\hline Cefoperazone & $49(66.2)$ & $38(77.6)$ & $16(69.6)$ & NT & NT \\
\hline Cefepime & $58(78.4)$ & $32(65.3)$ & $18(78.3)$ & $33(84.6)$ & $5(71.4)$ \\
\hline Imipenem & $16(21.6)$ & $4(8.2)$ & $5(21.7)$ & $10(25.6)$ & $5(71.4)$ \\
\hline Aztreonam & $54(73)$ & $29(59.2)$ & $8(34.8)$ & $27(69.3)$ & NT \\
\hline Gentamicin & $42(56.8)$ & $22(44.9)$ & $15(65.2)$ & 17 (43.6) & $5(71.4)$ \\
\hline Tobramycin & $37(50)$ & $20(40.8)$ & NT & $13(33.3)$ & $5(71.4)$ \\
\hline Amikacin & $23(31)$ & $7(14.3)$ & $9(39)$ & $12(30.8)$ & $6(85.7)$ \\
\hline Ciprofloxacin & $54(73)$ & 40 (81.6) & NT & NT & $5(71.4)$ \\
\hline Levofloxacin & $26(3)$ & $16(32.7)$ & $7(30.4)$ & $9(23)$ & $4(57.1)$ \\
\hline Chloramphenicol & $36(48.7)$ & $5(10.2)$ & $8(34.8)$ & NT & NT \\
\hline Tetracycline & 44 (59.5) & $18(36.7)$ & $16(69.6)$ & NT & 3 (42.9) \\
\hline
\end{tabular}

NT: Not tested; "Nitrofurantoin tested only with urine samples with \% resistant to Klebsiella spp. (33.3\%), E. coli (17.2\%), and Norfloxacin tested only with urine samples with \% resistant to Klebsiella spp. (50\%), and to Pseudomonas spp. (28.6\%) and to E. coli (72.5\%)

78.4\% of $K$. pneumoniae spp., $78.3 \%$ of Proteus spp., $71.4 \%$ of A. baumannii spp., and $65.3 \%$ of E. coli spp. (Table 3 ).

Carbapenems (imipenem) resistance was observed in $71.4 \%$ of $A$. baumannii spp., $25.6 \%, 21.7 \%$ of $P$. aeruginosa spp. and $21.6 \%$ of Proteus spp., Resistance to Ciprofloxacin was found in E. coli spp. (81.6\%), P. aeruginosa spp. (73\%) and A. baumannii spp. (71.4\%). Levofloxacin resistance was found in $57.1 \%$ of $P$. aeruginosa spp. and $35 \%$ of $K$. pneumoniae spp. (Table 3).

MRSA was $55.8 \%$, MR CoNS was $100 \%$, and VRE was $20 \%$. 
Citation: Hassan R, Gilany AHE, Elaal AMA, et al. (2021) Antibiotic Resistance Pattern of Bacteria Causing Hospital Acquired Infections in the New Mansoura General Hospital, Egypt. Arch Community Med 3(1):16-21

Table 4: Resistance patterns for isolated organisms.

\begin{tabular}{|l|l|l|l|}
\hline & Total isolates & \multicolumn{2}{c|}{ Resistant isolate $^{*}$} \\
\cline { 3 - 4 } & & Type of resistant & N (\%) \\
\hline S. aureus & 43 & MRSA & 24 (55.8) \\
\hline CoNS & 8 & Methicillin resistant & $8(100)$ \\
\hline Enterococci & 25 & VRE & $5(20)$ \\
\hline E. coli & 49 & Carbapenem resistant & $4(8.2)$ \\
\hline K. pneumoniae & 74 & CephR-Klebsiella & $63(85)$ \\
\hline Acinetobacter & & Carbapenem resistant & $16(21.6)$ \\
\hline P. aeruginosa & 7 & MDR & $7(100)$ \\
\hline
\end{tabular}

${ }^{*}$ From total isolates.

E. coli spp. resistance to carbapenem was $8.2 \%$. Ceph R-Klebsiella spp. was $85 \%$, and Carbapenem resistant $K$. pneumoniae spp. was $21.6 \%$. MDR-Pseudomonas spp. was $43.6 \%$ and MDR Acinetobacter spp. was $100 \%$ (Table 4).

\section{Discussion}

In this study $74.4 \%$ S. aureus spp. isolates was resistant to penicillin. CoNS exhibit $100 \%$ and $75 \%$ resistant to cefoxitin and penicillin; respectively. Enterococci spp. isolates were mostly resistant to ampicillin (88\%) and penicillin (80\%). A study in three teaching hospitals in North of Iran, reported that $100 \%$ S. aureus spp. was resistant to penicillin, cefoxitin and quinolones. CoNS were resistant to penicillin, cefoxitin and quinolones (100\%, 66\% and 100\%; respectively) [7]. In another study in Iranian hospital found that resistance to cefoxitin was $39.1 \%$, quinolones resistant $S$. aureus spp. was $39.1 \%$, and CoNS resistant to quinolones was $100 \%$. Enterococci spp. resistance to ampicillin was $57.1 \%$ and vancomycin resistance was $42.9 \%$ [8].

Although linezolid antibiotic considered from second line treatment, it exhibits resistance by $23 \% 12.5 \%$ and $16 \%$ of $S$. aureus spp., CoNS by $12.5 \%$, and Enterococci spp.; respectively. These results agreed with study in tertiary care University Hospital in India which revealed $77.5 \%$ of organisms are resistant to linezolid [9]. Although in another study in China, revealed no resistant to linezolid [10]. Linezolid should be used if there is resistant to vancomycin and not prescribed routinely, however some clinicians use it as first line of treatment without indication this could explain the arising resistance for it.

In this study there is increase in resistant of GNB to both third (ceftazidime, cefotaxime, and cefoperazone), and fourth generation cephalosporin (cefepime). The rate of resistance to these generations of cephalosporins varies worldwide. An Iranian study found that $39 \%$ of $P$. aeruginosa spp. were resistant to cefepime, and $68 \%$ are resistant to ceftazidime [11]. A study in Morocco, showed that $P$. aeruginosa spp. resistance to ceftazidime was $35.6 \%, K$. pneumoniae spp. resistance to ceftriaxone was $75.0 \%$ and to ceftazidime was $69.5 \%$, and $E$. Coli spp. resistance to ceftriaxone was $31.9 \%$ and to ceftazidime was $21.7 \%$ [12].

This study revealed that $71.4 \%, 25.6 \%, 21.7 \%$ and $21.6 \%$ of Acinetobacter spp., $P$. aeruginosa spp., Proteus spp., $K$. pneumoniae spp.; respectively were resistant to imipenem. A previous Egyptian study reported that resistance to imipenem was totally absent or very low [13]. Another study in three large tertiary care university hospitals in Egypt, Imipenem resistance was detected in $23.5 \%$ of $P$. aeruginosa spp. isolates and in $27.6 \%$ of $A$. baumannii spp. isolates [1]. Studies in Turkey, Italy, and France reported the presence of low levels of resistance to imipenem [14].

Quinolones antibiotics (ciprofloxacin and levofloxacin) exhibit resistance by different GNB as $81.6 \%, 73 \%$, and $71.4 \%$ of $E$. coli spp., K. pneumoniae spp., and Acinetobacter spp. were found to be Ciprofloxacin-resistant; respectively. Furthermore, 57.1\%, 35\%, 32.7\%, 30.4\%, and 23\% Acinetobacter spp., K. pneumoniae spp., E. coli spp., Proteus spp., and $P$. aeruginosa spp. were Levofloxacin resistant; respectively. A study in the National Cancer Institute, Egypt, E. coli spp. resistance to levofloxacin was $62.9 \%$ and ciprofloxacin resistance was $55.9 \%$. K. pneumoniae spp. resistance to levofloxacin was $30.7 \%$ and ciprofloxacin resistance was $36 \%$ [15]. Acinetobacter spp. and $E$. coli spp. showed the highest resistance to ciprofloxacin in an Iranian study [16].

There has been a dramatic increase in nosocomial infections due to antibiotic resistance and MDR pathogens throughout the world. MDR bacteria of concern include MRSA, VRE, $M D R P$. aeruginosa spp., and MDR A. baumannii spp. Most of the highprofile nosocomial organisms are MDR e.g. methicillin-resistant $S$. aureus spp. (MRSA). Multidrug-resistant-gram negative organisms (MDR-GNRs) have become increasingly prevalent in many hospitals [17]. In this study MRSA was $55.8 \%$, Methicillin resistant CoNS was $100 \%$. In a study at Assiut University Hospitals, Egypt stated that MRSA was $18.9 \%$ and CoNS methicillin resistance was $16 \%$ [18]. Worldwide, MRSA rates vary widely, from $1 \%$ in Denmark, Sweden, and The Netherlands to $40 \%$ in the United Kingdom, Greece, and Italy [19]. The US rate of $r 43$ was $52.9 \%$, and CoNS methicillin resistance was $76.6 \%$ [20]. VRE in this study was $20 \%$. This is similar to a study in Korea [21]. However much higher rate was reported in Iran (42.9\%) [8].

The high rate of GNP isolated from patients with nosocomial infections has a two clinical significance: A high prev- 
Citation: Hassan R, Gilany AHE, Elaal AMA, et al. (2021) Antibiotic Resistance Pattern of Bacteria Causing Hospital Acquired Infections in the New Mansoura General Hospital, Egypt. Arch Community Med 3(1):16-21

alence of MDR strains combined with limited therapeutic options; and a higher associated mortality, particularly for Gram-negative bacteraemia [22]. P. aeruginosa spp. had emerged in Egypt in recent years and seen mainly in nosocomial infections [23]. In this study MDR $P$. aeruginosa spp. was 43.6\%. This high rate of MDR has been reported in previous studies. In Menofia University Hospital MDR P. aeruginosa spp. was 52\% [24]. In another study in Egypt observed that MDR $P$. aeruginosa spp. was $36 \%$ [25]. On the other hand, in study in Iran detected that $30 \%$ of isolates were MDR with 100\% MDR A. baumannii spp. [26]. A multicenter study exhibited in in 46 ICUs in 11 Egyptian hospitals, Acinetobacter-MDR strains were $100 \%$ [27]. In the US National Healthcare Safety Network approximately $70 \%$ of Acinetobacter spp, was MDR [28]. In an Indian study multidrug resistance was seen in $63.3 \%$ of the $A$. baumannii spp. infections [29].

The high percentage of antimicrobial resistance in this study could be explained on the basis of prescription behaviors in the hospital as there is no established antibiotic policy; prescription has no standard regulation, overusing of empiric treatment without laboratory confirmation of susceptibility patterns and incomplete treatment courses with antibiotics.

\section{Study Limitations}

This is a single hospital study during a single year. Its results cannot be generalized to other hospital or during different periods. There is no breakdown of the resistance patterns by specific ward or department. Information from the specific departments may show specific strains dominating in those areas.

\section{Conclusions}

These results underline the need to integrate bacteria antibiotics sensitivity in all nosocomial infections surveillance system. Policies on the control of antibiotic usage have to be enforced and implemented to avoid the evolution of newer generations of pathogens with higher resistance, not only to the older generation drugs, but also to the relatively new ones. A nation-wide study is warranted to give the full picture of antibiotic resistance. Ongoing surveillance and test for antibiotic resistance will show the changing trends over time. These will help policy makers to develop a policy for antibiotic use in health care facilities.

\section{Funding}

None.

\section{Conflict of Interest}

None.

\section{Ethical considerations}

The study protocol was approved by IRB, Faculty of Medicine, Mansoura University as well as the Director of MNGH.

\section{References}

1. Saied T, Elkholy A, Hafez SF, et al. (2011) Antimicrobial resistance in pathogens causing nosocomial bloodstream infections in university hospitals in Egypt. Am J Infect Control 39: e61-e65.
2. El Kholy A, Baseem H, Hall GS, et al. (2003) Antimicrobial resistance in Cairo, Egypt 1999-2000: A survey of five hospitals. J Antimicrob Chemother 51: 625-630.

3. Lin MY, Weinstein RA, Hayden MK (2014) Multidrug-resistant organisms: Epidemiology and control. In: WR Javris, Bennet \& Brachman's hospital infections. Lippincott Williams \& Wolters Klumer Business, 181-207.

4. NHSN (2017) National Healthcare Safety Network (NHSN) patient safety components manual. CDC, Atlanta, Georgia.

5. CLSI (2017) M100: Performance standards for antimicrobial susceptibility testing. (27 $7^{\text {th }}$ edn), Clinical and Laboratory Standards Institute, Wayne, PA, USA.

6. NHSN (2016) National Healthcare Safety Network (NHSN) patient safety components manual. CDC, Atlanta, Georgia.

7. Behzadnia S, ADavoudi A, Rezai MS, et al. (2014) Nosocomial infections in pediatric population and antibiotic resistance of the causative organisms in north of Iran. Iran Red Crescent Med J 16: e14562.

8. Yaghoobi M, Arabestani M, Karami P (2018) The pattern of antibiotic resistance of common bacteria causing nosocomial infections. World Family Medicine 16: 177-183.

9. Mahendra M, Jayaraj BS, Lokesh KS, et al. (2018) Antibiotic prescription, organisms and its resistance pattern in patients admitted to respiratory ICU with respiratory infection in Mysuru. Indian J Crit Care Med 22: 223-230.

10. Zhang WJ, Wu CM, Wang Y, et al. (2011) The new genetic environment of cfr on plasmid pBS-02 in a Bacillus strain. J Antimicrob Chemother 66: 1174 -1175.

11. Esfahani BN, Basiri R, Mirhosseini SMM, et al. (2017) Nosocomial infections in intensive care unit: Pattern of antibiotic-resistance in Iranian community. Adv Biomed Res 6: 54.

12. Madani N, Rosenthal VD, Dendane T, et al. (2009) Health-care associated infections rates, length of stay, and bacterial resistance in an intensive care unit of Morocco: Findings of the International Nosocomial Infection Control Consortium (INICC). Int Arch Med 2: 29.

13. Saied GM (2006) Microbial pattern and antimicrobial resistance, a surgeon's perspective: Retrospective study in surgical wards and seven intensive-care units in two university hospitals in Cairo, Egypt. Dermatology 212: 8-14.

14. Spanu T, Luzzaro F, Perilli M, et al. (2002) Occurrence of extended-spectrum beta-lactamases in members of the family Enterobacteriaceae in Italy: Implications for resistance to beta-lactams and other antimicrobial drugs. Antimicrob Agents Chemother 46: 196-202.

15. Ashour HM, El-Sharif A (2009) Species distribution and antimicrobial susceptibility of gram-negative aerobic bacteria in hospitalized cancer patients. J Transl Med 7: 14.

16. Rajabi M, Abdar ME, Rafiei $\mathrm{H}$, et al. (2015) Nosocomial infections and epidemiology of antibiotic resistance in teaching hospitals in south east of Iran. Glob J Health Sci 8: 190-197.

17. Walsh TR, Toleman MA (2012) The emergence of pan-resistant gram-negative pathogens merits a rapid global political response. J Antimicrob Chemother 67: 1-3.

18. Ahmed SH, Daef EA, Badary MS (2009) Nosocomial blood stream infection in intensive care units at Assiut University Hospitals (Upper Egypt) with special reference to extended spectrum beta-lactamase producing organisms. BMC Res Notes 2: 76. 
Citation: Hassan R, Gilany AHE, Elaal AMA, et al. (2021) Antibiotic Resistance Pattern of Bacteria Causing Hospital Acquired Infections in the New Mansoura General Hospital, Egypt. Arch Community Med 3(1):16-21

19. Tiemersma EW, Bronzwaer SLAM, Lyytikainen O, et al. (2004) Methicillin-resistant staphyolococcus aureus in Europe, 19992002. Emerg Infect Dis 10: 1627-1634.

20. National Nosocomial Infections Surveillance System (2004) National nosocomial infections surveillance (NNIS) System report, data summary from January 1992 through June 2004, issued October 2004. Am J Infect Control 32: 470-485.

21. An H, Park S, Ko S (2017) Steady inflow of Vancomycin-Resistant Enterococci from outside a hospital. Korean J Health Assoc Infect Control Prev 22: 63-70.

22. Mayr FB, Yende S, Angus DC (2014) Epidemiology of severe sepsis. Virulence 5: 4-11.

23. Ali A, Abbasi S, Ahmed M (2009) Frequency of ES $\beta$ Ls producing nosocomial isolates in a tertiary care hospital in Rawalpindi. Pakistan Armed Forces Medical Journal 12: 23.

24. Mahmoud AB, Zahran WA, Hindawi GR, et al. (2013) Prevalence of multidrug-resistant Pseudomonas aeruginosa in patients with nosocomial infections at a university hospital in Egypt, with special reference to typing methods. Journal of Virology \& Microbiology 2013: 1-13.
25. Gad GF, El-Domany RA, Zaki S, et al. (2007) Characterization of Pseudomonas aeruginosa isolated from clinical and environmental samples in Minia, Egypt: Prevalence, antibiogram and resistance mechanisms. J Antimicrob Chemother 60: 10101017.

26. Zahra T, Moniri R. (2011) Detection of ESBLs and MDR in Pseudomonas aeruginosa in a tertiary-care teaching hospital. Iran J Clin Infect Dis 6: 18-23.

27. See I, Lessa FC, Abo ElAta O, et al. (2013) Incidence and pathogen distribution of healthcare-associated infections in pilot hospitals in Egypt. Infect Control Hosp Epidemiol 34: 1281-1288.

28. Sievert DM, Ricks P, Edwards JR, et al. (2013) Antimicrobial-resistant pathogens associated with healthcare-associated infections: Summary of data reported to the National Healthcare Safety Network at the Centers for Disease Control and Prevention, 2009-2010. Infect Control Hosp Epidemiol 34: 1-14.

29. Saravu K, Prasad M, Eshwara VK, et al. (2015) Clinico-microbiological profile and outcomes of nosocomial sepsis in an Indian tertiary care hospital - a prospective cohort study. Pathog Glob Health 109: 228-235. 